\title{
PERFORMANCE ANALYSIS OF A LIQUID COLUMN IN A CHEMICAL PLANT BY USING MPC
}

\author{
Babeeta Rathore ${ }^{1}$, Manju Khare ${ }^{2}$ \\ ${ }^{1}$ Pursuing M-Tech in Control System, Electrical \& Electronics Engg Dept, LNCT, Bhopal M.P., India \\ ${ }^{2}$ Assoc Prof, Electrical \& Electronics Engg Dept, LNCT, Bhopal M.P., India
}

\begin{abstract}
Model predictive control (MPC) is an advanced method of process control that has been in use in the process industries in chemical plants and oil refineries since the 1980s. In recent years it has also been used in power system balancing models. Model predictive controllers rely on dynamic models of the process, most often linear empirical models obtained by system identification. The main advantage of MPC is the fact that it allows the current timeslot to be optimized, while keeping future timeslots in account. This is achieved by optimizing a finite time-horizon, but only implementing the current timeslot. MPC has the ability to anticipate future events and can take control actions accordingly. PID and LQR controllers do not have this predictive ability. MPC is a digital control.
\end{abstract}

Keywords: Model predictive control (MPC); proportional-integral-derivative controller (PID)

\section{INTRODUCTION}

Liquid column Method is one of the best separations techniques in a chemical and petroleum industries, and tight control action is important from the plant economics. The purpose of the system is to separate a liquid mixture from two or more components. Liquid Column modelling and controlling both are very difficult because it's dealing with multivariable, nonlinear and non-stationary process. The composition control plays the vital role in liquid columns. Generally innovative process control tools increase the flexibility and performance of the chemical plants.

The conventional controller (PID) employed to control the liquid column does not guarantee tight control action because it's highly nonlinear. To solve critical control issues and to achieve better performance in industrial application, PID controllers are used but they face difficulties in controlling non-linear process and cannot predict immediate change in an input. To overcome these difficulties MPC controller is used.

\section{MODEL PREDICTIVE CONTROL}

Traditional feedback controllers operate by adjusting control action in response to a change in the output set point of a system. Model predictive control (MPC) is a technique that focuses on constructing controllers that can adjust the control action before a change in the output set point actually occurs. This predictive ability, when combined with traditional feedback operation, enables a controller to make adjustments that are smoother and closer to the optimal control action values. Below we see the basic structure of MPC:

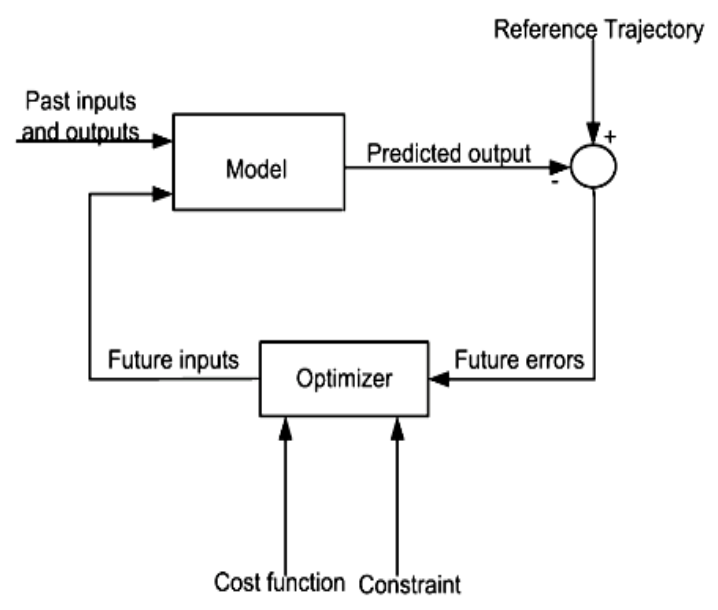

Fig 1: Basic structure of MPC

MPC consists of an optimization problem at each time instants, $\mathrm{k}$. The main point of this optimization problem is to compute a new control input vector to be feed to the system, and at the same time take process constraints into consideration (e.g. constraints on process variables).

\section{A BRIEF HISTORY OF INDUSTRIAL MPC}

This section presents an abbreviated history of industrial MPC technology. Figure 2 shows an evolutionary tree for the most significant industrial MPC algorithms, illustrating their connections in a concise way. 


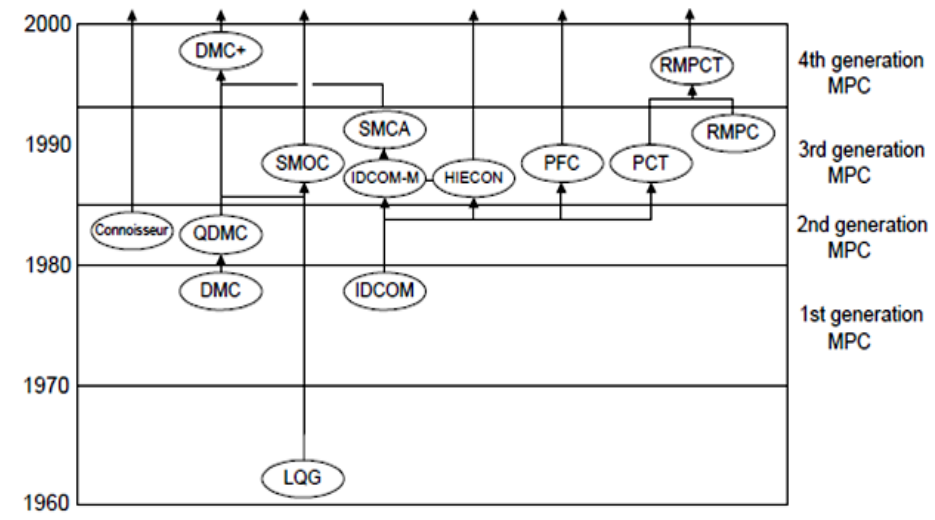

Fig 2: Approximate genealogy of linear MPC algorithms

Model predictive control (MPC) technique started to be implemented in industrial applications practically since 1970's. The studies related with MPC initialized with Richalet et al. (1978) as Model Predictive Heuristic Control (MPHC) technique and named as Model Algorithmic Control (MAC). The main characteristics of this type of controller include:

(1) The MPC controller attempts to optimize the process. Dynamic models representing how the Process behaves are used to predict future behavior and to determine the optimal operating point.

(2) The predictions of the future behaviour are also used to determine the control action taken by the controller. Disturbances can be rejected before they affect the process.

(3) MPC is a multi-input, multi-output (MIMO) controller. No input/output pairings need to be identified. All manipulated variables are moved simultaneously to control all the controlled variables. Interaction is accounted for with the dynamic models and used as an advantage in the control. Also, the number of inputs does not need to be equal to the number of outputs. MPC can handle non-square systems very easily.

\section{CONTROL OBJECTIVE FUNCTION OF MPC}

There are several objective functions but, we applied standard least-squares or quadratic programming objective function. QP formulation gives smoother control actions and the MPC will have more intuitive tuning parameters. The QP formulation is used in this thesis. The objective function is a sum of squares of the predicted errors (difference between the set points and the model-predicted outputs) and the control moves (changes in the control action from step to step).

A quadratic objective function for a predictive horizon of 3 and a control horizon of 2 can be written as.

$\varphi=Q\left(r_{k+1}-\hat{y}_{k+1}\right)^{2}+Q\left(r_{k+2}-\hat{y}_{k+2}\right)^{2}+Q\left(r_{k+3}-\hat{y}_{k+3}\right)^{2}+R \Delta u_{k}^{2}+R \Delta u_{k+1}^{2}$

Where $\hat{y}$ represents the model predicted outputs, $\mathrm{r}$ is the set point, $\Delta \mathrm{u}$ is the change in manipulated input from one sample time to the next, $\mathrm{Q}$ and $\mathrm{R}$ is a weight for the change in the output and manipulated input respectively.

For a prediction horizon of $\mathrm{P}$ and a control horizon $\mathrm{M}$, the least square objective function is written as.

$$
\varphi=Q \sum_{i=1}^{P}\left(r_{k+i}-\hat{y}_{k+i}\right)^{2}+R \sum_{i=0}^{M-1} \Delta u_{k+i}^{2}
$$

The optimization problem solved is usually stated as a minimization of the objective function, obtained by adjusting the $\mathrm{M}$ control moves.

One limitation of output weight is we cannot take null matrix. It can use step and impulse response data which can easily be obtained.

\subsection{MPC for MIMO Plants}

One advantage of an MPC Toolbox design (relative to classical multi-loop control) is that it generalizes directly to plants having multiple inputs and outputs. Moreover, the plant can be non-square, i.e., having an unequal number of actuators and outputs. Industrial applications involving hundreds of actuators and controller outputs have been reported.

\section{SYSTEM DESCRIPTION}

Figure 3 shows that the structure of a liquid column. The real time data are taken from by using a reflux rate of the column as kept constant and to give the sudden step changes of the Reboiler temperature. The black box models can be developed by correlating sequence relationship between input and output data, here the input is Reboiler temperature (manipulated variable) and the output is overhead product composition (controlled variable). After obtaining the data model has been developed by using a least square algorithm (LS). The main application of least squares is model fitting. Least square technique is mainly used for estimating the system parameter and minimization of error so that here the system parameter estimation and error minimization developed by least square method it is one of the system identification technique. 


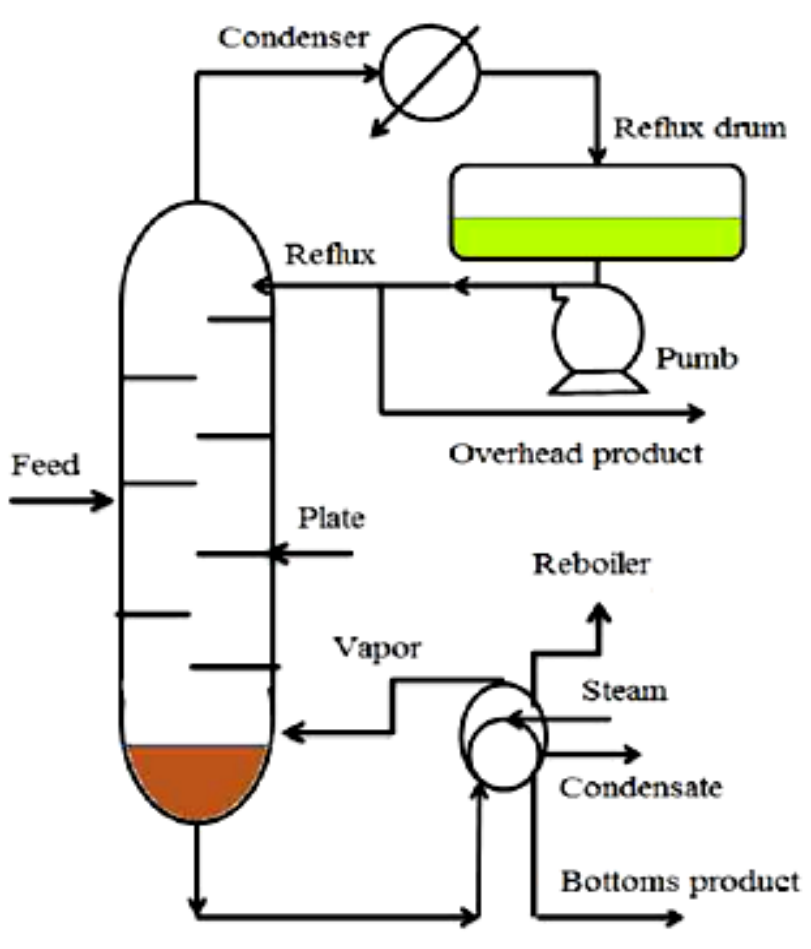

Fig 3: Structure of Liquid Column

\subsection{Modelling of Plant}

The models used for the prediction in adaptive control to be empirical input-output (Black-box) models. The main reason for this is that input/output measurement data are available for on-line estimation. These models fall under two major categories: deterministic and stochastic. Deterministic

Models give a complete description of the plant response. In practice these models tends to highly unrealistic since there typically exist aspect that are out of our control. Stochastic models contain some kind of random component usually defined on a probability space. In this thesis we deal with deterministic model. Let a system as a linear combination of past output, $\mathrm{y}(\mathrm{k})$, and past input, $\mathrm{u}(\mathrm{k})$

$$
y(k)+a_{1} y(k-1)+\ldots . .+a_{n} y(k-n)=b_{0} u(k)+b_{1} u(k-1)+\ldots . .+b_{n} u(k-m)
$$

Linear black box models can be obtained by ARX(Auto Regressive exogenous), ARMAX(Auto Regressive Moving Average exogenous) input etc.

\subsection{SIMULINK Model in MATLAB:}

Let we choose two variable w1 \& w2 which is manipulated outcomes of the two different controller PID and MPC. We assume liquid column is filling with a chemical whose level and concentration are too controlled by the controllers. Now for this we have to design a plant which has two input w1 and w2 also has two output level (h) and concentration $(\mathrm{Cb})$. The relation between input and output is

$$
\mathrm{Cb}=\int\left(\frac{\mathrm{w} 1(\mathrm{Cb} 1-\mathrm{Cb})}{\mathrm{h}}+\frac{\mathrm{w} 2(\mathrm{Cb} 2-\mathrm{Cb})}{\mathrm{h}}+\frac{\mathrm{Cb}}{(\mathrm{Cb}+1)^{2}}\right)
$$

$\mathrm{Cb} 1$ and $\mathrm{Cb} 2$ are the maximum and minimum concentration required for proper operation in liquid column. We choose $\mathrm{Cb} 1=24.9 \mathrm{M}$ and $\mathrm{Cb} 2=0.1 \mathrm{M}$.

$$
\mathrm{h}=\int_{1}^{\infty}(\mathrm{w} 1+\mathrm{w} 2-0.2 \sqrt{\mathrm{h}})
$$

Height of the liquid in column is calculated by the equation 1.2. By using these two equations we design the process model of the plant in MATLAB. Figure 4 shows the SIMULINK model of Process Plant named as chemical plant



Fig 4: SIMULINK Model of using control of chemical plant by PID or MPC

\section{RESULTS AND DISCUSSION}

A comparative analysis of the performance of the chemical plant by both MPC and PID controller is obtained by undergoing simulations in Matlab. The results show that MPC is better controller than PID.

MPC controllers are probably the best assumption that can be used for stable plants in the total absence of disturbance and measurement information.

At 0s delay: figure 5 to 8 shows the variation in concentration and level by PID or MPC. 




Fig 5: Concentration of liquid at 0s delays by PID controller

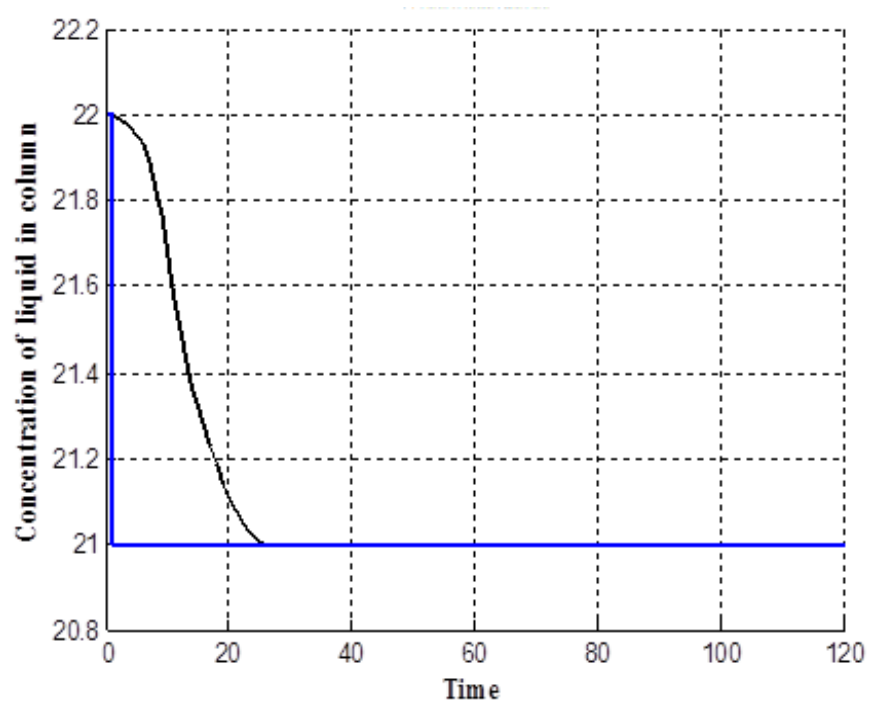

Fig 6: Concentration of liquid at 0s delays by MPC controller

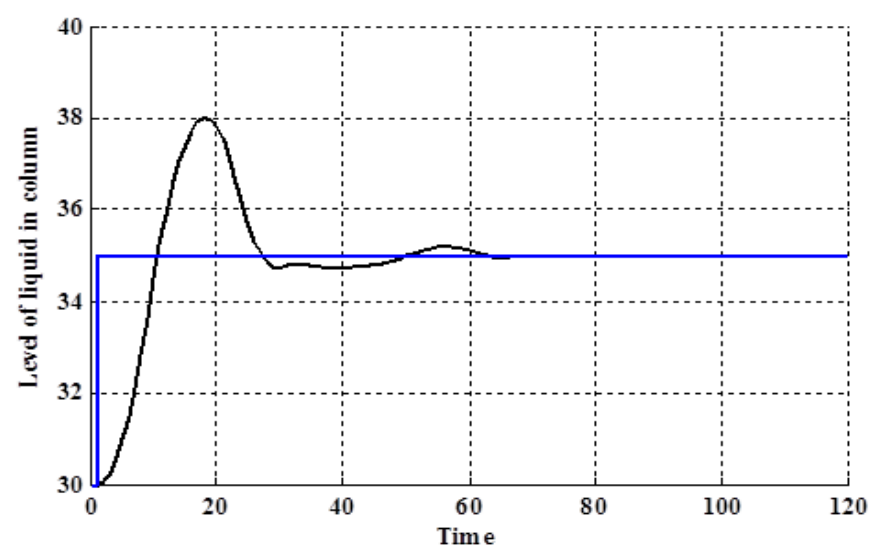

Fig 7: Level of Liquid in Column at 0s delay by PID Controller

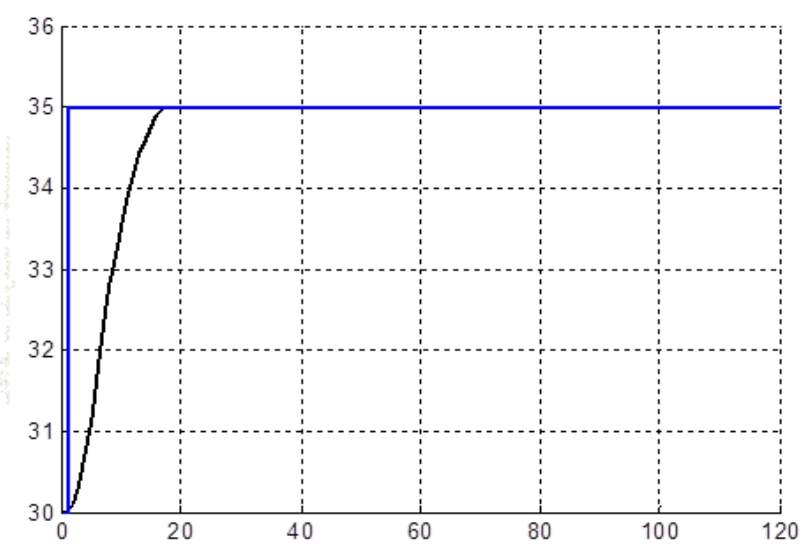

Fig 8: Level of Liquid in Column at 0s delay by MPC Controller

\section{CONCLUSIONS}

This Paper deals with the Model Predictive Controller and its working in process control. It also shows that by an example of liquid column it is the best methodology in advance process control. Based on the comparison of the two control methods, the process model MPC used to represent the system enables MPC controller to predict the state of the plant during the dynamic operation, which is particularly attractive as compared with PID because the dynamics change as the water level changes in the tanks, and a corresponding linearized model of the water tank system can be used in real time by the MPC.

\section{REFERENCES}

[1]. Allgower, F., \& Zheng, A., (Eds.). (2000). Nonlinear model predictive control, progress in systems and control theory. Vol. 26. Basel, Boston, Berlin: Birkhauser Verlag.

[2]. Bartusiak, R. D., \& Fontaine, R. W. (1997). Feedback method for controlling non-linear processes. US Patent 5682309.

[3]. Kouvaritakis, B., \& Cannon, M. (Eds.) (2001). Nonlinear predictive control, theory and practice. London: The IEE.

[4]. Maciejowski, J. M. (2002). Predictive control with constraints. Englewood Cliffs, NJ: Prentice Hall.

[5]. Martin, G., \& Johnston, D. (1998). Continuous modelbased optimization. In Hydrocarbon processing's process optimization conference, Houston, TX.

[6]. Young, R. E., Bartusiak, R. B., \& Fontaine, R. B. (2001). Evolution of an industrial nonlinear model predictive controller. In Preprints: Chemical process control-CPC VI, Tucson, Arizona

[7]. Mayne, D. Q., Rawlings, J. B., Rao, C. V., \& Scokaert, P. O. M. (2000). Constrained model predictive control: Stability and optimality.

[8]. MATLAB, Optimization and Control Toolbox, www.mathworks.com 